\title{
7,8-Dihydroxycoumarin inhibits A549 human lung adenocarcinoma cell proliferation by inducing apoptosis via suppression of Akt/NF- $\kappa$ B signaling
}

\author{
YUE WANG $^{1}$, CHANG-FENG LI ${ }^{2}$, LI-MING PAN ${ }^{3}$ and ZHONG-LI GAO ${ }^{1}$ \\ Departments of ${ }^{1}$ Thoracic Surgery and ${ }^{2}$ Endoscopy, China-Japan Union Hospital of Jilin University, Changchun, Jilin 133033; \\ ${ }^{3}$ Department of Pediatrics, The First Bethune Hospital of Jilin University, Changchun, Jilin 130021, P.R. China
}

Received January 14, 2013; Accepted April 5, 2013

DOI: $10.3892 /$ etm.2013.1054

\begin{abstract}
The Akt/NF- $\mathrm{AB}$ pathways are involved in numerous anti-apoptotic and drug-resistance events that occur in non-small cell lung cancer (NSCLC). In the present study, the role of 7,8-dihydroxycoumarin in the regulation of the anti-apoptotic Akt and NF- $\mathrm{Bp} 65$ signaling pathways was explored. A549 human lung adenocarcinoma cells were exposed to 7,8-dihydroxycoumarin with a final concentration of 25, 50 and $100 \mu \mathrm{mol} / \mathrm{l}$ for $48 \mathrm{~h}$. Quantitative polymerase chain reaction (PCR) and western blotting were performed to detect mRNA and protein expression, respectively. The MTT assay was performed to detect cell proliferation. The results demonstrated that anti-apoptotic phospho-Akt1 (pAkt1), phospho-IкB $\alpha(\mathrm{pI} \kappa \mathrm{B} \alpha), \mathrm{NF}-\kappa \mathrm{Bp} 65$ and Bcl-2 were inhibited and pro-apoptotic caspase-3 was upregulated in a concentration-dependent manner. At a concentration of $100 \mu \mathrm{mol} / 1$, the anti-apoptotic NF- $\kappa \mathrm{Bp} 65$ and Bcl-2 mRNA expression levels decreased 0.12 (5.82/48.5, treated/control)-fold and 0.17 (6.7/39.4, treated/control)-fold, respectively. The pro-apoptotic caspase-3 mRNA was upregulated 4.43 (39.4/8.9, treated/ control)-fold. The anti-apoptotic pAkt1, pI $\kappa \mathrm{B} \alpha, \mathrm{NF}-\kappa \mathrm{Bp} 65$ and $\mathrm{Bcl}-2$ proteins were downregulated, with blot grayscale values of 7.3 (vs. 52.4 control), 4.3 (vs. 42.2 control), 5.08 (vs. 44.5 control) and 5.92 (vs. 38.5 control), respectively. The proapoptotic caspase-3 was upregulated to a blot grayscale value of 27.8 (vs. 5.8 control). The proliferative activity of A549 cells was reduced significantly compared with that of the control cells (83.7, 27.2 and 9.5 vs. $100 \%$, respectively; $\mathrm{P}<0.05$ for each). 7,8-Dihydroxycoumarin plays an important role in the induction of apoptosis via suppression of $\mathrm{Akt} / \mathrm{NF}-\kappa \mathrm{B}$ signaling in A549 human lung adenocarcinoma cells in a
\end{abstract}

Correspondence to: Professor Zhong-Li Gao, Department of Thoracic Surgery, China-Japan Union Hospital of Jilin University, 126 Xiantai Street, Changchun, Jilin 130033, P.R. China

E-mail: zrlgaozl@sina.com

Key words: 7,8-dihydroxycoumarin, lung adenocarcinoma, apoptosis, signaling, Akt, NF-кB concentration-dependent manner. 7,8-Dihydroxycoumarin may be a candidate naturally-occurring drug for the treatment and prevention of lung adenocarcinoma.

\section{Introduction}

Naturally occurring coumarins, a group of plant-derived polyphenolic compounds, serve as antimitotic, immunomodulating, antiviral, anticancer and cytotoxic agents in humans (1,2). A coumarin derivative, 7,8-dihydroxycoumarin, an active plant lactone extracted from Daphne Korean Nakai (3), is mainly used as an analgesic, antibacterial and antiviral agent, as well as to prevent and treat liver fibrosis in the clinic (1).

7,8-Dihydroxycoumarin and analogs have demonstrated significant antitumor effects and promote tumor apoptosis (4-7) via multiple signaling pathways. Elinos-Báez et al reported that 7-hydroxycoumarin inhibits anti-apoptotic Bcl-2 expression in lung cancer cells and promotes the expression of pro-apoptotic Bcl-2-associated X protein (Bax) (8). Other studies identified that coumarin is able to induce cervical and colon cancer cell apoptosis by activating the mitochondrial pathway and the caspase-3-dependent apoptotic pathway, to downregulate the anti-apoptotic NF- $\kappa \mathrm{B}, \mathrm{Bcl}-2$ and $\mathrm{Bcl}-\mathrm{xL}$, and upregulate caspase- 3 to promote the release of cytochrome (cyt) $c(9,10)$. The coumarin derivative psoralidin is also able to enhance the role of tumor necrosis factor-related apoptosisinducing ligand (TRAIL) in promoting the apoptosis and necrosis of HeLa cervical cancer cells (11). Rasul et al reported that the coumarin derivative xanthoxyletin induces $\mathrm{S}$ phase arrest and apoptosis in SGC-7901 gastric cancer cells (12). Bhattacharyya et al demonstrated that 7-hydroxy-6-methoxycoumarin induces the downregulation of aryl hydrocarbon receptor (AhR), CYP1A1, proliferating cell nuclear antigen (PCNA), Stat-3, survivin, matrix metalloproteinase (MMP)-2, cyclin D1 and c-myc, and upregulation of p53, caspase-3 and tissue inhibitor of metalloproteinases (TIMP)-2 (13). Singh et al reported a coumarin derivative (RKS262) that inhibits the ovarian cancer cell cycle and promotes apoptosis in cancer cells (14). Additionally, the authors identified that the coumarin derivative upregulates pro-apoptotic proteins Bid and Bok and inhibits anti-apoptotic Bcl-xL and Mcl-1, independently of pro-apoptotic mitogen-activated protein 
kinase (MAPK) p38 and stress-activated protein (SAP)/c-Jun $\mathrm{N}$-terminal kinase (JNK) activation. Bhattacharyya et al reported that coumarin enhances pro-apoptotic p53, PCNA, Bad, Bax, apoptotic protease activating factor (Apaf), cyt $c$, caspase- 3 and caspase- 9 expression in melanoma (skin cancer) cells, and inhibits the anti-apoptotic factors Akt, Bcl-2, Bcl-xL and NF- $\mathrm{BB}(15)$. Thati et al also identified that coumarin derivatives enhance the malignancy of pro-apoptotic factors caspase-3 and -9 (16).

The main types of lung carcinoma include small-cell lung cancer (SCLC) and non-small-cell lung cancer (NSCLC); lung adenocarcinoma accounts for $40 \%$ of NSCLCs $(17,18)$. Lung adenocarcinoma cells overexpress multiple anti-apoptotic signals. The Akt/NF- $\mathrm{B}$ pathways are involved in a number of anti-apoptotic and drug-resistant events that occur in lung adenocarcinoma $(17,18)$. Therefore, we hypothesize that 7,8-dihydroxycoumarin may also play an important role in promoting the apoptosis of lung adenocarcinoma cells by suppressing the Akt and NF- $\kappa \mathrm{B}$ signaling pathways.

In the present study, 7,8-dihydroxycoumarin was administered to lung adenocarcinoma cells to investigate its effect on the apoptotic signaling pathways.

\section{Materials and methods}

Materials. 7,8-Dihydroxycoumarin (purity, 99.6\%; Tauto Biotech Ltd. Co., Shanghai, China) was dissolved in $0.9 \% \mathrm{NaCl}$ solution, followed by filtration with a $0.02-\mathrm{mm}$ filter (Millipore, Billerica, MA, USA). The structure of 7,8-dihydroxycoumarin is shown in Fig. 1. A total protein extraction kit and a TRIzol total RNA extraction kit were purchased from Invitrogen Life Technologies (Carlsbad, CA, USA). The anti-phospho-IкB $\alpha$ (phospho S32/S36; sc-8404), anti-NF-кBp65 (sc-8008), anti-Bcl-2 (sc-509) and anti-caspase-3 (sc-7272) antibodies were purchased from Santa Cruz Biotechnology, Inc. (Santa Cruz, CA, USA). The anti-phospho-Akt1 (phospho T308; ab105731) and anti-glyceraldehyde 3-phosphate dehydrogenase (GAPDH; ab8245) antibodies were purchased from Abcam (Beijing, China); these antibodies were mouse monoclonal. The horseradish peroxidase (HRP)-labeled goat anti-mouse secondary antibody was purchased from Abcam. 3-(4,5-Dimethylthiazol2-yl) 2,5-diphenyltetrazolium bromide (MTT) was purchased from Sigma (St. Louis, MO, USA). The Moloney murine leukemia virus reverse transcriptase (M-MLV RTase) kit was purchased from Promega Corporation (Shanghai, China). The 2X SYBR real-time polymerase chain reaction (PCR) kit was purchased from Roche Diagnostics (Shanghai, China). The bicinchoninic acid (BCA) protein detection kit and enhanced chemiluminescence (ECL) detection kit were purchased from Pierce Chemicals, Thermo Fisher Scientific Inc. (Rockford, IL, USA).

Cell line. The A549 human lung adenocarcinoma cell line was purchased from American Type Culture Collection (ATCC no. CCL-185; Manassas, VA, USA). The cells were cultured in Dulbecco's modified Eagle's medium (DMEM) containing $10 \%$ fetal bovine serum (Gibco, Invitrogen Life Technologies) in a $5 \% \mathrm{CO}_{2}$ incubator and passaged with $0.25 \%$ trypsin (Sigma, Ronkonoma, NY, USA) and $0.03 \%$ ethylenediamine tetraacetic acid (EDTA) solution.

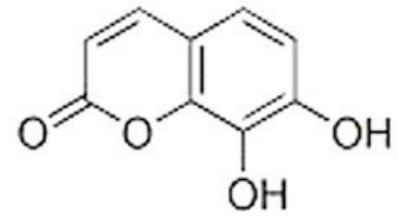

Figure 1. Structure of 7,8-dihydroxycoumarin.

Treatment. The A549 cells were digested, suspended and seeded into each well of six-well plates with a density of $1.0 \times 10^{6}$ cells $/ \mathrm{ml}$ in $2 \mathrm{ml}$ complete culture medium. The cells were cultured for $24 \mathrm{~h}$ and then exposed to 7,8-dihydroxycoumarin for 48 h. 7,8-Dihydroxycoumarin was dissolved in $0.9 \%$ $\mathrm{NaCl}$ solution and added to cells, forming a final concentration of 25,50 and $100 \mu \mathrm{mol} / 1$. Equivalent $0.9 \% \mathrm{NaCl}$ solution was added to cells as the control.

Quantitative PCR ( $q P C R)$. The A549 cells were harvested and total RNA was extracted with the total RNA extraction kit using the TRIzol method. The first strand cDNA was synthesized using M-MLV RTase according to the manufacturer's instructions. Real-time PCR was performed using the cDNA template according to the manufacturer's instructions. Amplification of GAPDH was used as an inner control in each reaction system. The reaction conditions were as follows: 40 cycles of $95^{\circ} \mathrm{C}$ for $30 \mathrm{sec}, 58^{\circ} \mathrm{C}$ for $60 \mathrm{sec}$ and $72^{\circ} \mathrm{C}$ for $60 \mathrm{sec}$. The primers were designed based on the Genbank sequence using Beacon Designer 7 (PREMIER Biosoft, Palo Alto, CA, USA). Primer synthesis and DNA sequencing were performed by Shanghai Sangon (China). The primer sequences were as follows: NF-кBp65, sense: 5'-GCAAAGGAAACGCCAGAAGC-3' and antisense: 5'- CACTACCGAACATGCCTCCAC-3'; Bcl-2, sense: 5'-ATGACTTCTCTCGTCGCTACT-3' and antisense: 5'-CCCATCCCTGAAGAGTTCCGA-3'; caspase-3, sense: 5'-CATGGCCTGTCAGAAAATAC-3' and antisense: 5'-TAACCCGAGTAAGAATGTGC-3'; GAPDH (housekeeper gene), sense: 5'-AATGTGTCCGTCGTGGATCTG-3' and antisense 5'-CAACCTGGTCCTCAGTGTAGC-3'.

Western blotting. Western blotting was used to detect the protein expression of phospho-Akt1 (pAkt1), phospho-IкB $\alpha$ $(\mathrm{pI} \kappa \mathrm{B} \alpha), \mathrm{NF}-\kappa \mathrm{Bp} 65, \mathrm{Bcl}-2$ and caspase-3. The A549 cells were harvested and cell lysis was performed using the eukaryotic cell lysis buffer followed by extraction of total protein, according to the manufacturer's instructions (Beyotime Inst. Biotech., Nanjing, Jiangsu, China). Protein quantity was determined by a BCA method. Using $30 \mu \mathrm{g}$ for each sample, proteins were separated by $12 \%$ sodium dodecyl sulfate polyacrylamide gel electrophoresis (SDS-PAGE) and were blotted with a wet transfer device (Bio-Rad, Shanghai, China) to a nitrocellulose membrane. The membrane was then immersed in blocking solution containing $10 \%$ skimmed milk in phosphate-buffered saline (PBS) Tween-20 (PBST), followed by agitation for $1 \mathrm{~h}$. After washing three times with Tris-buffered saline Tween-20 (TBST) for $5 \mathrm{~min}$ each, the membrane was immersed in primary antibody diluted to 1:1,000 in the blocking solution at room temperature and then agitated for $1 \mathrm{~h}$. After washing again, the membrane was incubated in HRP-labeled secondary antibody diluted to $1: 10,000$ in blocking solution at room 

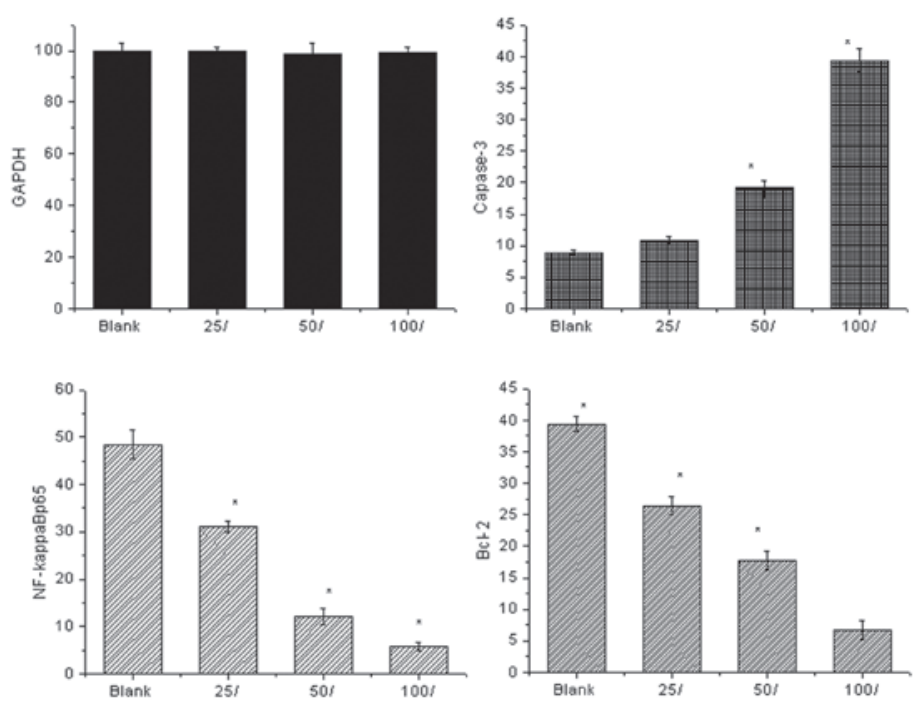

Figure 2. Relative mRNA levels (\%) vs. glyceraldehyde 3-phosphate dehydrogenase (GAPDH). 7,8-Dihydroxycoumarin concentrations: 25,50 and $100 \mu$ mol/1. Blank: control cells. GAPDH: housekeeper gene. As 7,8-dihydroxycoumarin was used in a series of dilutions, i.e. 25, 50 and $100 \mu$ mol/1, anti-apoptotic signaling was inhibited and pro-apoptotic signaling was activated. At $50.0 \mu \mathrm{mol} / 1$, the anti-apoptotic NF- $\mathrm{\kappa Bp} 65$ and Bcl-2 levels decreased 0.17 (8.9/52.6)-fold and 0.22 (9.5/42.3)-fold, respectively. Pro-apoptotic caspase-3 was upregulated 4.78 (38.7/8.1)-fold. ${ }^{*} \mathrm{P}<0.05$, compared with the control $(\mathrm{n}=3)$.

temperature and then agitated for $1 \mathrm{~h}$. After another rinse, the membrane underwent color development by an ECL method, followed by X-film photography. GAPDH protein was used as an inner control. The gray scale values (total raw density) of blots were measured with the VisionWorksLS analysis software available in the UVP EC3 (600) Imaging System (UVP, LLC, Upland, CA, USA).

MTT assay. After $48 \mathrm{~h}$, the medium was refreshed to discard the 7,8-dihydroxycoumarin. Cells were supplemented with $200 \mu \mathrm{l}$ MTT solution $(5 \mathrm{mg} / \mathrm{ml})$, followed by incubation in a $\mathrm{CO}_{2}$ incubator for another $4 \mathrm{~h}$. The supernatant was discarded and each well was supplemented with $500 \mu$ l dimethylsulfoxide (DMSO; Sigma). When the purple crystals at the bottom of the well were completely dissolved, the absorbance value was measured with a Thermo Multiskan MK3 microplate reader (Thermo Fisher Scientific Inc.,Waltham, MA, USA) at a wavelength of $490 \mathrm{~nm}$. Cell viability $(\%)=$ experimental absorbance/normal absorbance x100.

Statistical analysis. Data are presented as means \pm standard deviation (SD). The statistical software SPSS 13.0 (SPSS, Inc., Chicago, IL, USA) was used for statistical analysis. Paired comparisons were performed by the Student's t-test. $\mathrm{P}<0.05$ was considered to indicate a statistically significant difference.

\section{Results}

$m R N A$ levels detected by $q P C R$. Fig. 2 shows the expression levels of the cell signaling molecules detected by qPCR. Prior to 7,8-dihydroxycoumarin treatment, the control cells expressed high levels of anti-apoptotic NF-кBp65 and Bcl-2 mRNAs and a low level of pro-apoptotic caspase-3. As 7,8-dihydroxycoumarin was used in a series of dilutions $(25,50$ and $100 \mu \mathrm{mol} / \mathrm{l})$, the anti-apoptotic signaling was inhibited and the pro-apoptotic signaling was activated. The

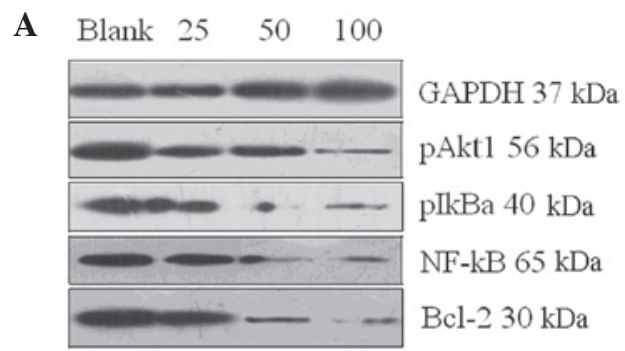

B Blank $25 \quad 50 \quad 100$

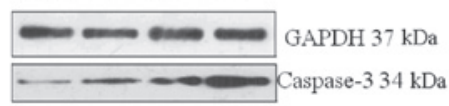

Figure 3. Blots of (A) anti-apoptotic and (B) pro-apoptotic proteins at $48 \mathrm{~h}$. 7,8-Dihydroxycoumarin concentrations: 25,50 and $100 \mu \mathrm{mol} / 1$; blank: control cells; glyceraldehyde 3-phosphate dehydrogenase (GAPDH): house-

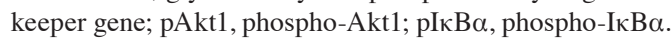

anti-apoptotic NF-кBp65 mRNA expression levels decreased 0.64 (31.04/48.5)-, 0.25 (12.1/48.5)- and 0.12 (5.82/48.5)-fold, respectively; and the levels of Bcl-2 mRNA decreased 0.67 (26.4/39.4)-, 0.45 (17.7/39.4)- and 0.17 (6.7/39.4)-fold, respectively. The pro-apoptotic caspase-3 increased 1.23 (10.9/8.9)-, 2.14 (19.1/8.9)-, and 4.43 (39.4/8.9)-fold, respectively. The pro-apoptotic induction effect of 7,8-dihydroxycoumarin is concentration-dependent.

Protein levels detected by western blotting. Fig. 3 shows the expression of the cell signaling molecules detected by western blotting. Prior to treatment with 7,8-dihydroxycoumarin, the control cells expressed high levels of anti-apoptotic pAkt1, $\mathrm{pI} \kappa \mathrm{B} \alpha, \mathrm{NF}-\kappa \mathrm{Bp} 65$ and $\mathrm{Bcl}-2$ proteins and a low-level of pro-apoptotic caspase-3 protein. As 7,8-dihydroxycoumarin was used in a series of dilutions $(25,50$ and $100 \mu \mathrm{mol} / \mathrm{l})$, the anti-apoptotic signaling was inhibited (Fig. 3A) and the pro-apoptotic signaling was upregulated (Fig. 3B). 
Table I. Gray scales of the western blots (48 h, \%/GAPDH).

7,8-Dihydroxycoumarin concentration $(\mu \mathrm{mol} / \mathrm{l})$

\begin{tabular}{lrrrr}
\cline { 3 - 4 } Protein blot & Cell control & 25 & 50 & 100 \\
\hline GAPDH $(37 \mathrm{kDa})$ & 100.30 & 101.40 & 99.50 & 102.20 \\
pAkt1 $(56 \mathrm{kDa})$ & 52.40 & 36.50 & 18.10 & 7.30 \\
pI & 42.20 & 13.70 & 7.60 & 4.30 \\
NF-kBp65 $(65 \mathrm{kDa})$ & 44.50 & 23.30 & 12.60 & 5.08 \\
Bcl-2 $(30 \mathrm{kDa})$ & 38.50 & 23.60 & 17.90 & 5.92 \\
Caspase-3 $(34 \mathrm{kDa})$ & 5.80 & 7.61 & 16.10 & 27.80
\end{tabular}

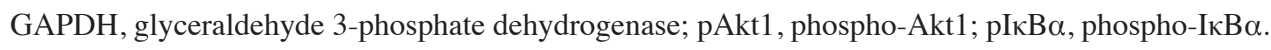

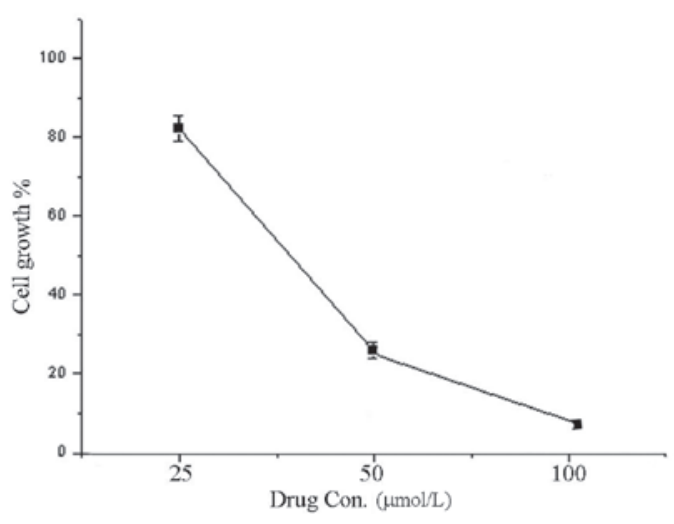

Figure 4. Cell proliferation of A549 lung adenocarcinoma cells treated with different concentrations of 7,8-dihydroxycoumarin $(25,50$ and $100 \mu \mathrm{mol} / \mathrm{l})$ for $48 \mathrm{~h}(\mathrm{n}=3)$.

Table I presents the complete gray scales of the blots shown in Fig. 3 to represent the total levels of the detected proteins. The anti-apoptotic pAkt1 protein blot grayscales were 36.5 , 18.1 and 7.3 vs. 52.4 (each 7,8-dihydroxycoumarin dose vs. control), respectively; the $\mathrm{pI} \kappa \mathrm{B} \alpha$ blot grayscales were 13.7, 7.6 and 4.3 vs. 42.2 , respectively; the $\mathrm{pNF}-\kappa \mathrm{Bp} 65$ blot grayscales were 23.3, 12.6 and 5.08 vs. 44.5; the Bcl-2 blot grayscales were 23.6, 17.9 and 5.92 vs. 38.5; and the pro-apoptotic caspase-3 blot grayscales were $7.61,16.1$ and 27.8 vs. 5.8 , respectively. The pro-apoptotic induction effect of 7,8-dihydroxycoumarin is concentration-dependent.

Cell proliferation. Fig. 4 illustrates cell viability at 48 h. The proliferative activity of A549 cells treated with 7,8-dihydroxycoumarin decreased and was significantly lower compared with that of the control cells $(83.7,27.2$ and 9.5 vs. $100 \%$, respectively; $\mathrm{P}<0.05$ for each). 7,8-Dihydroxycoumarin inhibited tumor cell proliferation in a concentration-dependent manner.

\section{Discussion}

Inhibition of the Akt/NF- $\mathrm{BB}$ pathways results in the upregulation of pro-apoptotic Fas/APO-1, FasL, Bax (17), caspase-8, caspase-3 and cyt $c$, with simultaneous downregulation of $\mathrm{NF}-\kappa \mathrm{B} \alpha, \mathrm{Akt}, \mathrm{Bcl}-2$ and Bcl-xL (18). In the present study, we used 7,8-dihydroxycoumarin to treat A549 lung adenocarcinoma cells and then performed qPCR and western blotting to detect the ability of 7,8-dihydroxycoumarin to change the levels of anti-apoptotic pAkt, $\mathrm{pI} \kappa \mathrm{B} \alpha, \mathrm{pNF}-\kappa \mathrm{B}$ p 65 and $\mathrm{Bcl}-2$, as well as pro-apoptotic caspase-3.

Prior to treatment with 7,8-dihydroxycoumarin, there is an overexpression of Akt1 phosphorylated in control cells (19). Hyperactivated pAktl has serine-threonine protein kinase activity and triggers the cascaded enzymes, resulting in an increased phosphorylation of $\mathrm{I} \kappa \mathrm{B} \alpha$ at serines 32 and 36. $\mathrm{pI} \kappa \mathrm{B} \alpha$ was disassociated from the $\mathrm{I} \kappa \mathrm{B} \alpha / \mathrm{NF}-\kappa \mathrm{B}$ complex, resulting in a release of $\mathrm{pNF}-\kappa \mathrm{B}$ causing an increase of $\mathrm{NF}-\kappa \mathrm{Bp} 65$ at the mRNA and protein levels. The hyperactivated pAkt1 also causes anti-apoptotic Bcl-2 to be maintained at high mRNA and protein levels, resulting in the sustained proliferation of the A549 control cells.

The use of 7,8-dihydroxycoumarin to treat A549 cells resulted in a marked downregulation of pAkt1 and $\mathrm{pI} \kappa \mathrm{B} \alpha$, as well as NF- $\mathrm{Bp} 65$ at the mRNA and protein levels. The downregulation of pAkt1 indicates that the serine-threonine protein kinase activity of Akt was reduced. Subsequently, the phosphorylation of $\mathrm{I} \kappa \mathrm{B} \alpha$ was reduced. Thus the $\mathrm{I} \kappa \mathrm{B} \alpha / \mathrm{NF}-\kappa \mathrm{B}$ complex inhibited the release of $\mathrm{NF}-\kappa \mathrm{B}$, resulting in a reduction in $\mathrm{NF}-\kappa \mathrm{Bp} 65$ levels. As the serine-threonine protein kinase activity of Akt was reduced, anti-apoptotic Bcl-2 was simultaneously downregulated, so that the suppression of the apoptosis of A549 cells was reduced; therefore, apoptosis was facilitated.

The downregulation of pAkt1 and NF-кBp65 demonstrated that the signal amplification and transduction pathways were efficiently suppressed. Accordingly, the pro-apoptotic caspase-3 expression was increased. As reported in previous studies, upregulated caspase-3 inhibits IKK2 $(20,21)$ in necrotized or apoptotic cancer cells, resulting in a further reduction in the phosphorylation of $\mathrm{I} \kappa \mathrm{B} \alpha$, causing the NF- $\kappa \mathrm{Bp} 65$ level to be further reduced. The upregulated caspase-3 also directly inhibits the NF- $\mathrm{NBp} 65$ protein (22), causing a secondary downregulation of NF- $\mathrm{NBp} 65$ in apoptotic cancer cells. Therefore, the NF- $\kappa$ Bp65 signaling was markedly suppressed in A549 cells in the present study. The apoptotic A549 cells were observed to undergo reduced proliferation. The MTT assay results also demonstrated that the proliferation of A549 cells was significantly inhibited by 7,8-dihydroxycoumarin. In 
addition, the pro-apoptotic induction effect of 7,8-dihydroxycoumarin was concentration-dependent.

In conclusion, 7,8-dihydroxycoumarin inhibits the proliferation of A549 human lung adenocarcinoma cells and induces their apoptosis via $\mathrm{Akt} / \mathrm{NF}-\kappa \mathrm{B}$ signaling suppression in a concentration-dependent manner. Akt and NF- $\kappa \mathrm{B}$ may be targets for the treatment of lung adenocarcinoma. 7,8-Dihydroxycoumarin may be a candidate naturally occurring drug for the treatment and prevention of lung adenocarcinoma.

7,8-Dihydroxycoumarin, as an extract of naturally occurring plants, is safe and has a high efficacy. Therefore, it may be used in the clinic to treat lung carcinoma.

\section{References}

1. Kontogiorgis C, Detsi A and Hadjipavlou-Litina D: Coumarin-based drugs: a patent review (2008 - present). Expert Opin Ther Pat 22: 437-454, 2012.

2. Anand P, Singh B and Singh N: A review on coumarins as acetylcholinesterase inhibitors for Alzheimer's disease. Bioorg Med Chem 20: 1175-1180, 2012.

3. Chakraborty AK, Funasaka Y, Pawelek JM, Nagahama M, Ito A and Ichihashi M: Enhanced expression of melanocortin-1 receptor (MC1-R) in normal human keratinocytes during differentiation: evidence for increased expression of POMC peptides near suprabasal layer of epidermis. J Invest Dermatol 112: 853-860, 1999

4. Yang EB, Zhao YN, Zhang K and Mack P: Daphnetin, one of coumarin derivatives, is a protein kinase inhibitor. Biochem Biophys Res Commun 260: 682-685, 1999.

5. Finn GJ, Creaven BS and Egan DA: Daphnetin induced differentiation of human renal carcinoma cells and its mediation by p38 mitogen-activated protein kinase. Biochem Pharmacol 67: 1779-1788, 2004.

6. Finn GJ, Kenealy E, Creaven BS and Egan DA: In vitro cytotoxic potential and mechanism of action of selected coumarins using human renal cell lines. Cancer Lett 183: 61-68, 2002.

7. Riveiro ME, Moglioni A, Vazquez R, Gomez N, Facorro G, Piehl L, de Celis ER, Shayo C and Davio C: Structural insights into hydroxycoumarin-induced apoptosis in U-937 cells. Bioorg Med Chem 16: 2665-2675, 2008

8. Elinos-Báez CM, León F and Santos E: Effects of coumarin and $7 \mathrm{OH}$-coumarin on bcl-2 and Bax expression in two human lung cancer cell lines in vitro. Cell Biol Int 29: 703-708, 2005.

9. Chuang JY, Huang YF, Lu HF, Ho HC, Yang JS, Li TM, Chang NW and Chung JG: Coumarin induces cell cycle arrest and apoptosis in human cervical cancer HeLa cells through a mitochondria- and caspase-3 dependent mechanism and NF-kappaB down-regulation. In Vivo 21: 1003-1009, 2007.
10. Saidu NE, Valente S, Bana E, Kirsch G, Bagrel D and Montenarh M: Coumarin polysulfides inhibit cell growth and induce apoptosis in HCT116 colon cancer cells. Bioorg Med Chem 20: 1584-1593, 2012.

11. Bronikowska J, Szliszka E, Jaworska D, Czuba ZP and Krol W: The coumarin psoralidin enhances anticancer effect of tumor necrosis factor-related apoptosis-inducing ligand (TRAIL). Molecules 17: 6449-6464, 2012.

12. Rasul A, Khan M, Yu B, Ma T and Yang H: Xanthoxyletin, a coumarin induces S phase arrest and apoptosis in human gastric adenocarcinoma SGC-7901 cells. Asian Pac J Cancer Prev 12: 1219-1223, 2011.

13. Bhattacharyya SS, Paul S, Dutta S, Boujedaini $\mathrm{N}$ and Khuda-Bukhsh AR: Anti-oncogenic potentials of a plant coumarin (7-hydroxy-6-methoxy coumarin) against 7,12-dimethylbenz [a] anthracene-induced skin papilloma in mice: the possible role of several key signal proteins. Zhong Xi Yi Jie He Xue Bao 8: 645-654, 2010.

14. Singh RK, Lange TS, Kim KK and Brard L: A coumarin derivative (RKS262) inhibits cell-cycle progression, causes pro-apoptotic signaling and cytotoxicity in ovarian cancer cells. Invest New Drugs 29: 63-72, 2011.

15. Bhattacharyya SS, Paul S, Mandal SK, Banerjee A, Boujedaini N and Khuda-Bukhsh AR: A synthetic coumarin (4-methyl-7 hydroxy coumarin) has anti-cancer potentials against DMBA-induced skin cancer in mice. Eur J Pharmacol 614: 128-136, 2009.

16. Thati B, Noble A, Creaven BS, Walsh M, McCann M, Devereux M, Kavanagh K and Egan DA: Role of cell cycle events and apoptosis in mediating the anti-cancer activity of a silver(I) complex of 4-hydroxy-3-nitro-coumarin-bis(phenanthroline) in human malignant cancer cells. Eur J Pharmacol 602: 203-214, 2009.

17. Hsu YL, Kuo PL and Lin CC: Proliferative inhibition, cell-cycle dysregulation, and induction of apoptosis by 7,8-dihydroxycoumarin in human non-small cell lung cancer A549 cells. Life Sci 75: 2303-2316, 2004.

18. Li Y, Xing D, Chen Q and Chen WR: Enhancement of chemotherapeutic agent-induced apoptosis by inhibition of NF-kappaB using 7,8-dihydroxycoumarin. Int J Cancer 127: 462-473, 2010.

19. Jang BC: The fruit juice of Morinda citrifolia (noni) downregulates HIF-1 $\alpha$ protein expression through inhibition of $\mathrm{PKB}$, ERK-1/2, JNK-1 and S6 in manganese-stimulated A549 human lung cancer cells. Int J Mol Med 29: 499-504, 2012.

20. Barkett M and Gilmore TD: Control of apoptosis by Rel/NF-кB transcription factors. Oncogene 18: 6910-6924, 1999.

21. Tang G, Yang J, Minemoto Y and Lin A: Blocking caspase-3 mediated proteolysis of IKK $\beta$ suppresses TNF- $\alpha$-induced apoptosis. Mol Cell 8: 1005-1016, 2001.

22. Ravi R, Mookerjee B, van Hensbergen Y, Bedi GC, Giordano A, El-Deiry WS, Fuchs EJ and Bedi A: p53-mediated repression of nuclear factor-kappaB RelA via the transcriptional integrator p300. Cancer Res 58: 4531-4536, 1998. 